\title{
Novel Insight Into the Molecular and Metabolic Mechanisms Orchestrating IL-17 Production in $\gamma \delta$ T Cells
}

\begin{abstract}
Xu Chen ${ }^{1,2+}$, Samantha Morrissey ${ }^{2,3+}$, Fuxiang Chen ${ }^{1,4 *}$ and Jun Yan ${ }^{2 *}$
${ }^{1}$ Department of Clinical Immunology, Shanghai Ninth People's Hospital, Shanghai Jiao Tong University School of Medicine, Shanghai, China, ${ }^{2}$ Immuno-Oncology Program, Division of Immunotherapy, Department of Surgery, James Graham Brown Cancer Center, University of Louisville, Louisville, KY, United States, ${ }^{3}$ Department of Microbiology and Immunology, School of Medicine, University of Louisville, Louisville, KY, United States, ${ }^{4}$ Faculty of Medical Labratory Science, Shanghai Jiaotong University School of Medicine, Shanghai, China
\end{abstract}

Increasing evidence has demonstrated that IL-17-producing $\gamma \delta T$ cells ( $\gamma \delta \mathrm{T} 17)$ play a tumor-promoting role in a series of cancers via various mechanisms in mice and human cancers, though the relationship between $\gamma \delta \mathrm{T} 17$ and human tumors has yet to be extensively characterized and established. Molecular signals such as intrinsic cascade, environmental cues and cellular metabolic pathways including nutrient uptake and utilization in $\gamma \delta \mathrm{T} 17$ cells are significantly important for their activation, differentiation, and function. Understanding the molecular mechanisms and metabolic pathways of $\gamma \delta T 17$ cells in both the physiological setting and tumor environment would contribute to the development of therapeutic approaches or drugs targeting $\gamma \delta \mathrm{T} 17$ for immunotherapy in cancers.

Keywords: innate immune cells, $\gamma \delta$ T17 cells, transcriptional regulation, metabolic reprogramming, cancer immunotherapy

Innate $\gamma \delta \mathrm{T}$ cells are a complex cohort of cells with diverse functionality in both physiological and disease conditions. While $\gamma \delta \mathrm{T}$ cells can be subdivided into multiple different subsets based on expression profile, they can largely be classified into two main functional groups- IFN- $\gamma$ producing $(\gamma \delta \mathrm{T} 1)$ and IL-17-producing $(\gamma \delta \mathrm{T} 17) \gamma \delta \mathrm{T}$ cells (1). While both direct and indirect antitumor effects of $\gamma \delta \mathrm{T}$ cells have been reported, the emerging consensus within the field suggests that the $\gamma \delta \mathrm{T} 17$ subset possess pro-tumorigenic characteristics mainly mediated by IL-17A production. Specifically, IL-17A from $\gamma \delta \mathrm{T}$ cells has been shown to induce angiogenesis within the tumor microenvironment (TME), and increase recruitment of immunosuppressive cell types like myeloid-derived suppressor cells (MDSCs), neutrophils, and tumor associated macrophages (2). Therefore, given the prominent role of IL-17 in tumorigenesis, it is important to better understand the mechanisms responsible for regulating IL-17 secretion in cancer and at baseline in normal physiological conditions. Here, we review the mechanistic drivers and metabolic pathways controlling IL-17 production in $\gamma \delta$ T cells in hopes to provide new approaches to cancer treatment by targeting $\gamma \delta \mathrm{T} 17$.

\section{$\gamma \delta$ T17 IN PHYSIOLOGICAL CONDITIONS AND TUMOR ENVIRONMENT}

$\gamma \delta \mathrm{T}$ cells are a unique subset of cells that combine conventional $\mathrm{T}$ cell adaptive immune features with rapid innate-like responses. Given this specific functionality, $\gamma \delta \mathrm{T}$ cells, particularly $\gamma \delta \mathrm{T} 17$, 
are often found in barrier and mucosal sites like the skin, oral mucosa, gut lamina propria, and lung in both mice and humans (3-7). Murine $\gamma \delta \mathrm{T} 17$ have been shown to play important roles in tissue homeostasis, anti-infectious pathogen clearance and body temperature maintenance despite an overall low abundance in the body $(3,4,8)$. Furthermore, in mice, $\gamma \delta \mathrm{T} 17$ cells contain $\mathrm{V} \gamma 4^{+}, \mathrm{V} \gamma 6^{+}$and minor $\mathrm{V} \gamma 1^{+}$subsets, and characteristically express ROR $\gamma \mathrm{t}$, c-Maf, and CCR6 (5-7, 9, 10). While both $\mathrm{V} \gamma 4^{+}$and $\mathrm{V} \gamma 6^{+}$subsets have been shown to produce IL-17, $\mathrm{V} \gamma 6^{+}$cells produce IL-17 exclusively, and augment production in the context of cancer or inflammation whereas $\mathrm{V} \gamma 4^{+}$are more heterogeneous and can produce IFN- $\gamma$ or IL-17 depending upon environmental context. The difference in cytokine profile is likely a result of differential development and peripheral regulation. It has been shown that development of $\mathrm{V} \gamma 6^{+}$is restricted to a functional embryonic wave that is dependent on the fetal thymus whereas ${\mathrm{V} \gamma 4^{+}}^{+}$are more complex and are heavily reliant on bone marrow progenitors (5). Further studies revealed differential homing patterns in the dermis and lung, and to a less extent in the lymph nodes (LN) and spleen, between the two subsets with $\mathrm{V} \gamma 6^{+}$often outcompeting $\mathrm{V} \gamma 4^{+}$. However, in spontaneous and transplantable cancer models, both subsets have been identified as prominent IL-17 producers $(2,11,12)$. While the protumoral characteristics of $\gamma \delta \mathrm{T} 17$ cells are not the topic of this review it should be noted that other prominent tumor promoting roles include functioning like regulatory $\mathrm{T}(\mathrm{Treg}) / \mathrm{T}$ helper2 (Th2) like cells, interfering with dendritic cell effector functioning, and inhibiting $\mathrm{T}$ cell effector functioning via the programmed death-1 (PD-1)-programmed death ligand-1 (PD-L1) pathway $(2,13,14)$.

In humans, $\gamma \delta \mathrm{T}$ cells mainly consist of tissue-resident $\mathrm{V} \delta 1^{+}$ and peripheral $\mathrm{V} \delta 2^{+}$subsets. Tissue-resident $\mathrm{V} \delta 1^{+} \gamma \delta \mathrm{T}$ cells are often found in epithelial layers and play significant roles against infections and tissue integrity (15). The $\mathrm{V} \delta 2^{+}$(mostly $\mathrm{V} \gamma 9^{+}$) population is more heterogeneous and can respond to a variety of pathogens (16). Both of these subsets can produce IFN$\gamma$ upon activation, but the $\mathrm{V} \delta 1^{+}$is reported to produce more IL-17 in some cancers like pancreatic ductal adenocarcinoma and colorectal carcinoma despite limited overall characterization studies $(16,17)$. Like their murine counterparts, human $\gamma \delta \mathrm{T} 17$ cells have been shown to promote tumor growth in a variety of human cancers including colorectal cancer, lung cancer, breast cancer and pancreatic ductal adenocarcinoma. (13, 16, 18, 19). However, a recent study revealed that breast-resident $\mathrm{V} \delta 1^{+}$cells differentially produce IFN- $\gamma$, not IL-17, and that breast-resident ${\mathrm{V} \delta 1^{+}}^{+}$are associated with remission in triple-negative breast cancer (20), thus demonstrating the complexity of human $\gamma \delta$ T17. There are some conserved protumor effectors mechanisms between murine and human $\gamma \delta$ T17 cells including increased angiogenesis and inhibition of $\alpha \beta \mathrm{T}$ cells, but other distinct mechanisms have been identified in humans as shown in Figure 1 (2). While further characterization of the role $\mathrm{V} \delta 1^{+}$play within the human tumor microenvironment of different cancers is warranted, a limited collection of evidence suggests these cells convey an immunosuppressive pro-tumoral phenotype.

Overall, $\mathrm{V} \gamma 6^{+}$and to an extent $\mathrm{V} \gamma 4^{+}$cells in mice and $\mathrm{V} \delta 1^{+}$cells in some human cancers like colorectal carcinoma and pancreatic ductal adenocarcinoma are considered to be tumor progressing $\gamma \delta$ T17. However, further studies are needed for exploring the human $\gamma \delta \mathrm{T} 17$ and its relationship with other cancer types. The molecular mechanisms governing IL-17 production are often specific to each particular cell type and will be covered individually in the next section. Therefore, understanding the molecular mechanisms and metabolic pathways orchestrating $\gamma \delta$ T17 cells would help contribute to developing new immunotherapies in cancers.

\section{MOLECULAR SIGNALS ORCHESTRATING IL-17 PRODUCTION IN $\gamma \delta$ T CELLS}

Cytokine production in $\gamma \delta \mathrm{T}$ cells can be preprogrammed in the embryonic thymus or induced in the periphery. Either way, cytokine production in $\gamma \delta \mathrm{T}$ cells requires complex networking. As shown in Figure 2, IL-17 production in murine $\gamma \delta \mathrm{T}$ cells is controlled by various transcriptional factors and also regulated by external stimulations. In this next section, we will identify specific transcription factors associated with IL-17 production in $\gamma \delta \mathrm{T} 17$.

\section{Transcription Factors (TFs)}

One of the most prominent transcription factors associated with IL-17 production is ROR $\gamma \mathrm{t}$. It is known to control IL17 production in Th17 cells and similarly has been found to be a core transcription factor for IL-17 production in murine $\mathrm{CD}^{-} 7^{-} \gamma \delta \mathrm{T}$ cells $(1,21)$. However, unlike Th17 cells, ROR $\gamma \mathrm{t}$ expression alone in $\gamma \delta$ cells is enough to induce IL-17 whereas Th17 cells often need collaborative signals from TGF- $\beta$, IL-6, or IL-21. Interestingly, ROR $\gamma \mathrm{t}$ can maintain its own expression by binding at the Rorc CNS +10 (a conserved non-coding sequence located $10 \mathrm{~kb}$ from the $\operatorname{Rorc}(\mathrm{t})$ transcription start site) (10). ROR $\gamma$ t usually cooperates with other TFs such as STAT3, IRF4, and BATF in Th17 differentiation (22), but what specific factors regulate $\mathrm{ROR} \gamma \mathrm{t}$ in the context of $\gamma \delta \mathrm{T} 17$ cells remains largely unexplored.

One TF that has been identified to augment ROR $\gamma$ t driven IL17 production in $\gamma \delta$ T cells is c-Maf. The AP- 1 TF c-Maf has been found to predominantly bind at Rorc CNS +10 , thus stabilizing ROR $\gamma$ t expression (10). c-Maf also inhibits the binding of TCF1 (a negative regulator of ROR $\gamma \mathrm{t}$ ) to Rorc to further support the expression of ROR $\gamma$ t. Furthermore, c-Maf regulates chromatin accessibility thus increasing the probability of ROR $\gamma \mathrm{t}$ binding. Interestingly, c-Maf can also promote $\gamma \delta$ T17 through a ROR $\gamma \mathrm{t}-$ independent mechanism via directly regulating Blk and Tcf7 (encoding TCF1).

It has been reported that IRF4, ROR $\alpha$ and BATF are not required in IL-17 production of $\gamma \delta \mathrm{T}$ cells $(22,23)$, but a recent study revealed that IRF4 played a significant role in IL17 production of murine dermal $\gamma \delta \mathrm{T}$ cells including $\mathrm{V} \gamma 4^{+}$ and ${\mathrm{V} \gamma 6^{+}}^{+}$subsets (24). Specifically IRF4 links IL-1R and IL23R signaling pathways to IL-17 production. One of the main transcription factors upstream from IRF4 is STAT3 (24). STAT3 activation is crucial for ROR $\gamma$ t expression in Th17 cells and also significant for IL-17 production in $\gamma \delta \mathrm{T}$, though some subsets 


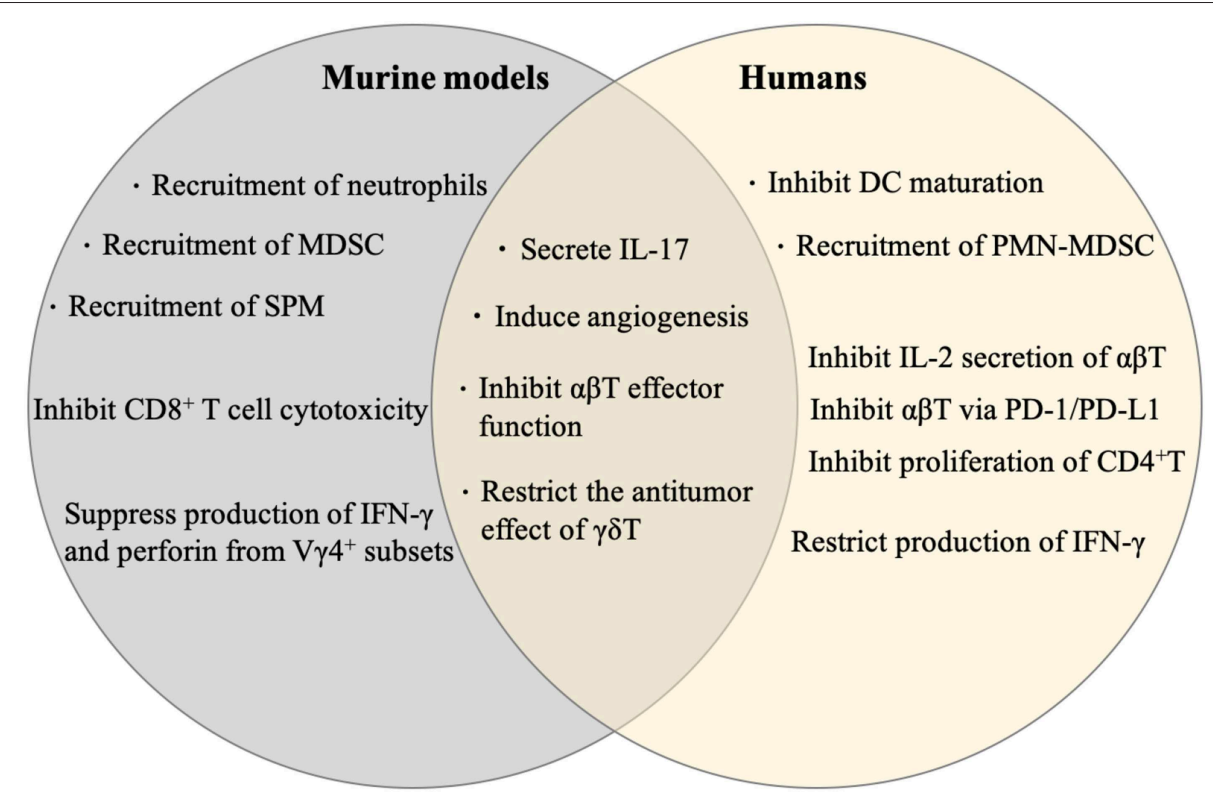

FIGURE 1 | Proposed mechanisms (differential and shared pro-tumor mechanisms) by $\gamma \delta$ T17 in murine models and humans (2, 11-13, 16, 18, 19).

of $\gamma \delta$ T have been found to be independent of STAT3 (24-26). Specifically, IL-23-induced STAT3 signaling plays a pivotal role in the production of IL-17 in dermal $\mathrm{V} \gamma 4^{+}$but not in $\mathrm{V} \gamma 6^{+}$ subsets (24). Similar to STAT3, some other TFs have been found to control IL-17 production in $\gamma \delta \mathrm{T}$ subsets. The high-mobility group (HMG) TFs SOX4 and SOX13 were required for $\mathrm{V} \gamma 4^{+}$ subsets, and SOX4 was an essential regulator of Rorc whereas SOX13 regulated Blk expression (27). Moreover, their upstream TF HEB (HeLa E-box binding protein) regulated the expression of SOX4 and SOX13 by interacting with the regulatory region of DNA ( $25 \mathrm{~kb} 5^{\prime}$ of the Sox 4 transcriptional start site and predicted $32 \mathrm{~kb} 5^{\prime}$ of the second start site of the Sox 13 locus) (28). The promyelocytic leukemia zinc finger (PLZF) TF was required for IL-17 secretion and maturation in $\mathrm{V} \gamma 6^{+}$subsets, but the detailed molecular mechanism remains to be explored (29). Interestingly, a recent report found that $\mathrm{PLZF}^{+} \gamma \delta \mathrm{T}$ cells promote a thermogenic response via directly producing cytokines such as IL-17 and TNF- $\alpha$ and indirectly maintaining catecholamine sensitivity (8). Collectively, apart from the core transcription factor ROR $\gamma \mathrm{t}$, universal transcription factors (cMaf, IRF4), which play a role in all $\gamma \delta \mathrm{T} 17$ subsets, and partial transcription factors (STAT3, HEB, SOX4, SOX13, and PLZF), which play a role in some $\gamma \delta \mathrm{T} 17$ subsets, work in concert or independently for the controlling of IL-17 production in different $\gamma \delta$ T subsets.

\section{Cell Surface Receptors and Cellular Intrinsic Cascade}

Mouse $\gamma \delta$ T17 cells express a variety of innate receptors including TLR1, TLR2, and dectin-1, but not TLR4. Activation of TLRs and dectin-1 leads to increased IL-17 production in $\gamma \delta \mathrm{T}$ cells (30), solidifying their role as non-histocompatibility complex restricted lymphocytes. Moreover, $\gamma \delta$ T17 cells express IL$23 \mathrm{R}$ and IL-1R which, following IL-1 $\beta$ with IL-23 stimulation, enhances IL-17 gene expression and protein production (6, 31, 32), suggesting that both PAMP and cytokine receptors play significant roles in the IL-17 production in $\gamma \delta \mathrm{T}$ cells. In fact, the indispensable roles of IL-1R and/or IL-23R in $\gamma \delta$ T17-mediated diseases such as experimental autoimmune encephalomyelitis (EAE) and psoriasis-like skin inflammation have been validated in murine models $(6,31,32)$. By exploring the molecular mechanism underlying the IL-1 $\beta$-IL-17 axis, IL1R-MyD88-mTORC2 was found in both dermal $\mathrm{V} \gamma 4^{+}$and $\mathrm{V} \gamma 6^{+}$ subsets, which primarily produced IL-17 (24). MyD88 is an adaptor protein which is required for most TLR signaling and therefore is necessary for TLR signaling-induced expansion and cytokine production of $\gamma \delta$ T17. However, the detailed cascade or mechanism of TLR signaling in IL-17 production of $\gamma \delta$ $\mathrm{T}$ remains to be understood. The cytokine IL-23 differentially enhanced IL-17 production via the IL-23R/STAT3/IRF4 pathway in dermal ${\mathrm{V} \gamma 4^{+}}^{+}$and via the IL-23R/RelA/IRF4 pathway in dermal $\mathrm{V} \sigma^{+}(24)$. These results suggested that IL-1 $\beta$ and IL-23 synergistically induced IL-17 production albeit through distinct pathways. Unexpectedly and of note, IL-17 itself is a negative regulator of $\gamma \delta \mathrm{T} 17$ as $I l-17 r$ knockout increased the IL-17 production of $\gamma \delta \mathrm{T}$ from cervical LN and inguinal $\operatorname{LN}(5,33)$. The mechanism behind this negative feedback loop have yet to be determined.

Furthermore, both the classical and non-canonical NF- $\kappa$ B signaling pathways are important for $\gamma \delta$ T17. RelA or RelB conditional deficiency leads to reduction of $\gamma \delta$ T17 cells through reducing $\mathrm{Il}-17$ and Rorc expression at the transcriptional level, and p52, not p50 was also required for IL-17 production (34). NF$\kappa \mathrm{B}$-inducing kinase $(\mathrm{NIK})$, which is required for non-canonical NF- $\kappa \mathrm{B}$ signaling, was essential for IL-17 production as NIK 


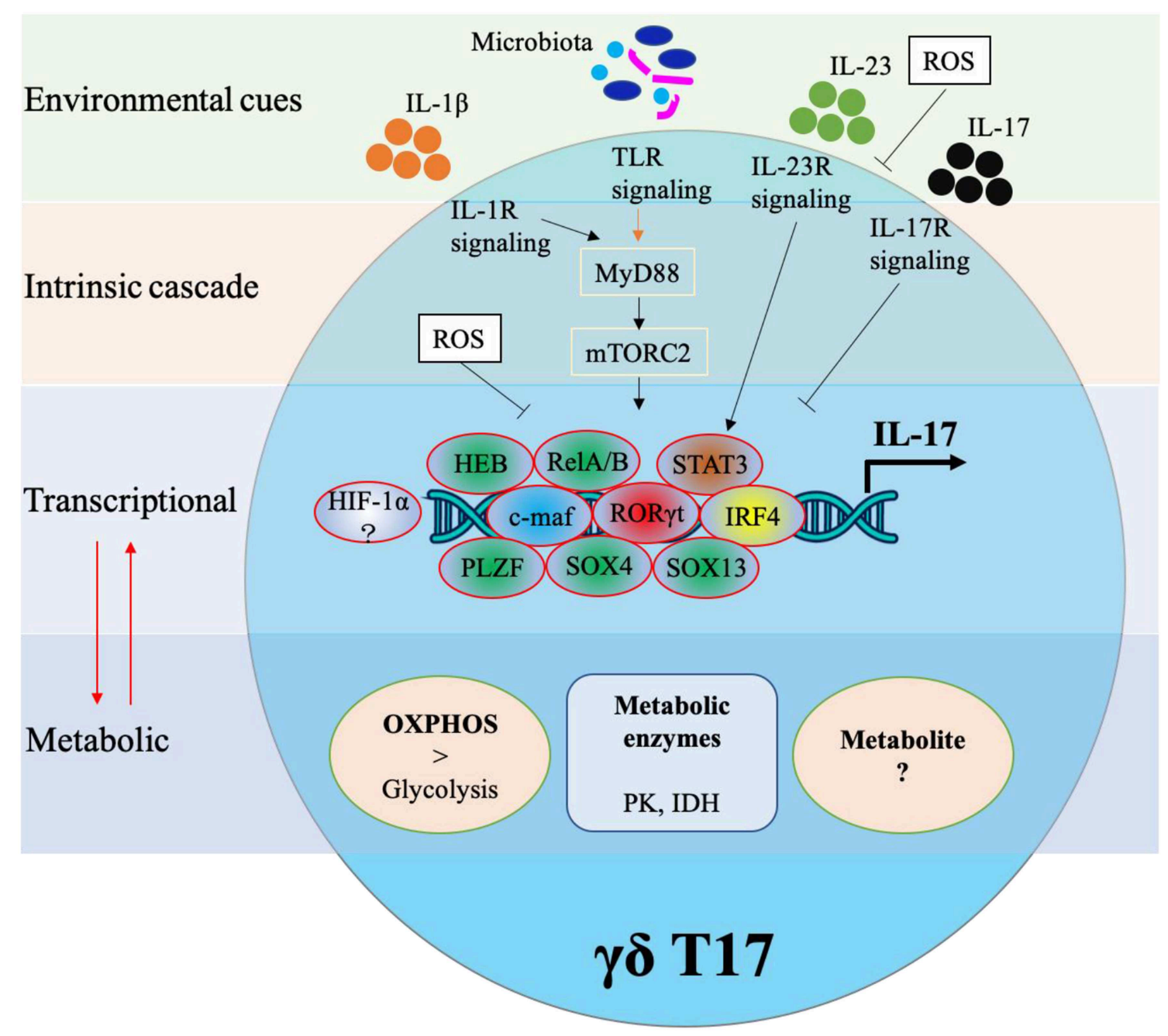

FIGURE 2 | Transcriptional and metabolic regulation of IL-17 production in murine $\gamma \delta$ T cells.

depletion led to impaired Rorc and Sox13 expression (35). Notch signaling and its downstream target Hes1, one of the basic helix-loop-helix (bHLH) proteins, were essential for the IL-17producing function of mature $\gamma \delta \mathrm{T}$ cells in the periphery (36). It should be noted that the majority of mouse $\gamma \delta$ T17 cells get functional preprogramming in the thymus, and factors like Notch signaling that have influence on the development of $\gamma \delta$ T17 may affect the IL-17 production of $\gamma \delta$ T cells.

It has been reported that TCR signaling is a major determinant of the functional differentiation of $\gamma \delta \mathrm{T}$ cells in the thymus. Strong TCR signaling determines the lineage fate of the earliest progenitor T cells toward the $\gamma \delta$ subset $(37,38)$. However, little is known about how $\gamma \delta$ TCR engagement drives IL-17 production in mature $\gamma \delta$ T cells. A recent study identified the Syk/PI3K/Akt pathway as one means to control the development of $\gamma \delta \mathrm{T} 17$ (39). Additionally, c-Maf regulation of ROR $\gamma t$ which was noted above is dependent on $\gamma \delta$ TCR ligation with too strong a signal limiting c-Maf expression (10). Overall, a variety of signaling pathways including PAMPS, cytokines, Notch and $\gamma \delta$ TCR ligation have been shown to orchestrate IL-17 production in murine $\gamma \delta$ T cells.

It is important to keep in mind that human $\gamma \delta \mathrm{T} 17$ are different from murine $\gamma \delta$ T17. Little is known about human $\gamma \delta \mathrm{T} 17$ in terms of origin, differentiation, and transcriptional regulation. While they express ROR $\gamma \mathrm{t}$, CCR6, IL-23R, and IL$1 \mathrm{R}$ like their murine counterparts (40), human $\gamma \delta \mathrm{T} 17$ cells may originate from naive precursors in the peripheral blood when activated with pathogen products and IL-23 stimulation (41). STAT3 deficiency in humans leads to a loss of IL-17 in $\gamma \delta$ $\mathrm{T}$ cells though it does not affect the frequency of $\gamma \delta \mathrm{T}$ cells (42). ROR $\gamma$ t inhibition could selectively target IL-17-producing innate cells including $\gamma \delta$ T17 in patients with spondyloarthritis, demonstrating that ROR $\gamma$ t antagonism could be a promising therapeutic approach (43). It has been reported that a cocktail of cytokines could maintain and differentiate human $\gamma \delta \mathrm{T} 17$ $(44,45)$, however a detailed mechanism has not been elucidated. Therefore, more information is needed for a better understanding 
of the mechanism underlying the development and function of IL-17-producing $\gamma \delta \mathrm{T}$ cells in humans.

\section{CELLULAR METABOLISM AND METABOLIC REPROGRAMMING OF $\gamma \delta$ T17 CELLS}

Cellular metabolism is coming to the forefront as an important indicator of cellular function. Specifically, cells often undergo metabolic reprogramming in the context of disease which ultimately affects the cells natural effector function. Naive $\mathrm{T}$ cells, for example, are quiescent with low metabolic demands and mainly rely on oxidative phosphorylation (OXPHOS) for ATP, while activated $\mathrm{T}$ cells dramatically upregulate glycolysis and downregulate mitochondria-dependent fatty acid oxidation (FAO) and pyruvate oxidation through the TCA cycle (46). This hypermetabolic reprogramming meets the demands for $\mathrm{T}$ cell activation in the context of infection or autoimmunity. Conversely in cancer, exhausted $\mathrm{T}$ cells are often found to be in a hypometabolic state which corresponds to their overall suppressed phenotype. The relationship between metabolism and $\mathrm{T}$ cell function has been summarized in detail elsewhere (4749). However, how metabolism impacts the effector function and differentiation of $\gamma \delta \mathrm{T}$ cells has been largely unexplored. Here, we will review what is known about the factors orchestrating $\gamma \delta$ T17 cell metabolism and how they may contribute to the understanding of $\gamma \delta \mathrm{T}$ cell metabolism for immunotherapy.

\section{Metabolic Pathway and Cellular Function in $\gamma \delta \mathrm{T}$ Cells}

Just like their cytokine profiles, the metabolic signatures of $\gamma \delta$ $\mathrm{T}$ cells differ between the two main subsets. $\gamma \delta \mathrm{T} 1$ cells have a preference for glycolytic metabolism while $\gamma \delta \mathrm{T} 17$ rely more on OXPHOS (24). Classically, this fits with the pattern that pro-inflammatory cells rely more heavily on glycolysis while anti-inflammatory/pro-tumoral cells mainly utilize OXPHOS or fatty acid metabolism. Inhibition of glycolysis with 2-deoxyD-glucose (2-DG) showed no impact on the IL-17 production of dermal $\gamma \delta \mathrm{T}$, while inhibition of isocitrate dehydrogenase (IDH) or pyruvate kinase (PK) significantly reduced IL-17 production of dermal $\gamma \delta \mathrm{T}$ cells in a dose-dependent manner (24), suggesting that the citric acid cycle (TCA) and OXPHOS play a significant role in IL-17 production. One of the key regulatory enzymes that serves as a central mediator between extrinsic signals like cytokines and environmental cues to cell-intrinsic metabolism is mammalian/mechanistic target of rapamycin (mTOR) $(24,50-52)$. There are two distinct mTOR complexes, mTORC1 and mTORC2 which contain scaffold proteins Raptor or Rictor, respectively. In dermal $\gamma \delta \mathrm{T}$ cells, stimulation with IL-1 $\beta$ and/or IL-23 directly activates mTOR resulting in increased IL-17 production. However, conditional knockout (cKO) of Rictor leads to reduced IL-17 production in both $\mathrm{V} \gamma 4^{+}$and ${\mathrm{V} \gamma 6^{+}}^{+}$subsets while Raptor cKO has no influence on IL-17 production of dermal $\gamma \delta \mathrm{T}$ (24). Neither Raptor cKO nor Rictor cKO impacted 2NBDG uptake in T-cell indicating that mTOR signaling specifically impacts OXPHOS.
Overall, these results suggest differential roles of mTORC1 and mTORC2 in the regulation of $\gamma \delta$ T17. Rictor cKO alone leads to more dysfunctional mitochondria and reactive oxygen species (ROS) production, which implies that functionally respiring mitochondria are essential to $\gamma \delta$ IL-17 production. Additionally, ROS negatively feeds back to IL-17 production in $\gamma \delta \mathrm{T}$ cells since treatment with ROS inhibitor N-acetyl-L-cysteine (NAC) rescued the IL-17 production (24). A recent report found that murine $\mathrm{V} \gamma 6^{+} \gamma \delta \mathrm{T} 17$ cells displayed low expression of ROS neutralizing antioxidant glutathione. Consequently, tumorassociated neutrophils were then able to inhibit the proliferation of murine $\mathrm{V} \gamma 6^{+} \gamma \delta \mathrm{T} 17$ cells via induction of ROS, suggesting novel approach for targeting the neutrophil/ROS/ $\gamma \delta \mathrm{T} 17$ axis in the tumor microenvironment (53).

While OXPHOS is most often mentioned in the context of metabolism and ATP generation, a secondary less recognized function is thermogenesis, or the generation of body heat. Uncoupling of OXPHOS from ATP synthesis via uncoupling protein 1 (UCP1) results in the potential energy of the electron transport chain being converted into thermal energy or heat. A recent study found that $\gamma \delta$ T17 cells play an important role in regulating this process (8). $\gamma \delta \mathrm{T}$ cells and IL-17 have been linked to maintaining homeostasis through $\mathrm{T}$ regulatory cell $\left(\mathrm{T}_{\text {reg }}\right)$ mediated thermogenesis. $\mathrm{T}_{\text {regs }}$ dominate the $\mathrm{CD} 4^{+}$ compartment within adipose tissue and generate large amounts of IL-33, a cytokine critical for body temperature regulation. They found that ${\mathrm{V} \gamma 6^{+}}$accumulate in adipose tissue over time and can directly influence non-shivering thermogenesis. Specifically, the $\mathrm{CD}_{27}-\gamma \delta \mathrm{T}$ cells through IL-17 mediate expansion of adipocyte resident $\mathrm{T}_{\text {regs }}$ which secrete the IL-33 necessary to induce UCP1 thermogenic effector function. Mice deficient in $\mathrm{V} \gamma 6^{+}$or global IL-17KOs were unable to successfully regain thermostatic equilibrium following cold challenge indicating the importance of $\gamma \delta$ generated IL-17 in thermogenesis.

The generation of IL-17 in $\gamma \delta \mathrm{T}$ cells is closely tied with mitochondria function and oxidative phosphorylation capacity. It has been shown that $\gamma \delta \mathrm{T} 17$ cells preferentially utilize OXPHOS over glycolysis and reduction in OXPHOS capacity correlates with decreased mitochondria function and concomitantly decreased IL-17 production. Furthermore, decreased IL-17 and ${\mathrm{V} \gamma 6^{+}}^{+}$cells in adipose tissue results in decreased uncoupling of OXPHOS and overall inability to thermoregulate in response to environmental thermofluctuations. However, further investigation into how OXPHOS and TCA metabolism regulates IL-17 production is warranted. It remains unclear how pre-programming in the thymus affects metabolism, whether there are any differences in metabolic condition between activated $\gamma \delta \mathrm{T} 17$ and resting $\gamma \delta \mathrm{T} 17$, and how $\gamma \delta \mathrm{T} 17$ metabolism differs from Th17 cells. Previous studies have shown that TF Myc is critical for glycolysis and glutaminolysis in activated T-cells, and HIF$1 \alpha$ is important for Th17 differentiation $(50,54)$. How these transcription factors impact $\gamma \delta \mathrm{T} 17$ development, particularly in the context of a hypoxic tumor microenvironment, has yet to be explored. In summary, limited information about the metabolism of $\gamma \delta \mathrm{T} 17$ has been reported. With the advance of new technologies such as systems metabolomics and single cell 
sequencing, more details on the metabolism of $\gamma \delta$ T17 could be revealed.

\section{CROSSTALK BETWEEN $\gamma \delta$ T17 AND OTHER CELLS AND MICROORGANISM IN TME}

We are just beginning to acquire a better understanding of the molecular mechanisms and metabolic regulations governing $\gamma \delta$ T17. However, the question becomes even more complex when taking into account the interaction between $\gamma \delta \mathrm{T} 17$, other cells, and microorganisms in the TME. A recent report characterizing the interaction between microbiota, immune cells and $\gamma \delta \mathrm{T} 17$ in lung adenocarcinoma microenvironment found that altered commensal microbiota promote lung cancer by activating $\gamma \delta$ T17 through PMAP/MyD88-mediated production of IL-1 $\beta$ and IL-23 from myeloid cells. (18). These tumor-associated $\gamma \delta \mathrm{T} 17$ showed a distinct transcriptional profile from that of spleen indicating environmental context is important for dictating function. This report builds on the previous suggestion that intact commensal microbiota and $\gamma \delta \mathrm{T}_{17}{ }^{+}$in the lungs are necessary to mount sufficient immune responses against B16/F10 or LLC tumor challenge (55). Therefore, further investigation into the drivers behind altered lung commensal microbiota is warranted along with identification of pathologic species to help tease apart the role of $\gamma \delta \mathrm{T} 17$ in health and disease. Whether the tumor-associated $\gamma \delta \mathrm{T} 17$ display a different metabolic condition as compared to other $\gamma \delta \mathrm{IL}-17$ producing subsets remains unknown. In murine models, oral microbiota drives $\gamma \delta \mathrm{T} 17$ proliferation and activation via $\mathrm{CD}_{103}{ }^{+}$DCs' cell-to-cell contact (33), while in the human colorectal cancer microenvironment, $\gamma \delta \mathrm{T} 17$ were polarized by microbiota-activated inflammatory DC-producing IL-23 and played a pivotal role in the cancer progression (16). A key tumor promoting phenotype of IL-17 is to recruit tumor-associated neutrophils or PMN-MDSCs to the

\section{REFERENCES}

1. Ribot JC, deBarros A, Pang DJ, Neves JF, Peperzak V, Roberts SJ, et al. CD27 is a thymic determinant of the balance between interferon-gamma- and interleukin 17-producing gammadelta T cell subsets. Nat Immunol. (2009) 10:427-36. doi: 10.1038/ni.1717

2. Fleming C, Morrissey S, Cai Y, Yan J. gammadelta T Cells: unexpected regulators of cancer development and progression. Trends Cancer. (2017) 3:561-70. doi: 10.1016/j.trecan.2017.06.003

3. Krishnan S, Prise IE, Wemyss K, Schenck LP, Bridgeman HM, McClure FA, et al. Amphiregulin-producing gammadelta $\mathrm{T}$ cells are vital for safeguarding oral barrier immune homeostasis. Proc Natl Acad Sci USA. (2018) 115:1073843. doi: $10.1073 /$ pnas. 1802320115

4. Dejima T, Shibata K, Yamada H, Hara H, Iwakura Y, Naito S, et al. Protective role of naturally occurring interleukin-17A-producing gammadelta $\mathrm{T}$ cells in the lung at the early stage of systemic candidiasis in mice. Infect Immun. (2011) 79:4503-10. doi: 10.1128/IAI.05799-11

5. Haas JD, Ravens S, Duber S, Sandrock I, Oberdorfer L, Kashani E, et al. Development of interleukin-17-producing gammadelta $\mathrm{T}$ cells is restricted to a functional embryonic wave. Immunity. (2012) 37:4859. doi: 10.1016/j.immuni.2012.06.003 local microenvironment. However, tumor-associated neutrophils can inhibit $\gamma \delta \mathrm{T} 17$ via ROS production (53). With more emerging reports, the crosstalk between $\gamma \delta \mathrm{T} 17$, microbiota and other cells reveals a complicated, dynamic network. Focusing on the regulatory factors driving IL-17 transcription in $\gamma \delta \mathrm{T}$ cells can help elucidate their role in cancer and could be the key to targeting $\gamma \delta$ T17-mediated tumor growth and progression.

\section{CONCLUDING REMARKS}

$\gamma \delta$ T17 promote cancer progression through IL-17 via various mechanisms in murine models and some human cancers. Understanding the molecular and metabolic mechanisms orchestrating IL-17 production of $\gamma \delta \mathrm{T}$ cells provides us with a better understanding of how these cells are supposed to function in health and how disease alters these processes. Knowing the intricate mechanisms governing IL-17 production can help contribute to the development of new therapies targeting $\gamma \delta$ T17-associated inflammation and tumor progression.

\section{AUTHOR CONTRIBUTIONS}

$\mathrm{XC}$ and SM drafted the manuscript. JY and FC discussed and revised the manuscript. All authors read and approved the final manuscript for submission.

\section{FUNDING}

This work was supported by the NIH R01CA213990 and R01AI128818 (JY) and by the National Natural Science Foundation of China 81870762 (FC); XC was supported by the China Scholarship Council (CSC No. 201806230234), Shanghai Sailing Program (19YF1427500), Shanghai Municipal Commission of Health and Family Planning (No. 20184Y0025), and Shanghai Ninth People's Hospital affiliated to Shanghai Jiao Tong University School of Medicine (No. JYZZ013).
6. Cai Y, Shen X, Ding C, Qi C, Li K, Li X, et al. Pivotal role of dermal IL17-producing gammadelta $\mathrm{T}$ cells in skin inflammation. Immunity. (2011) 35:596-610. doi: 10.1016/j.immuni.2011.08.001

7. Li Y, Wu J, Luo G, He W. Functions of Vgamma4 T cells and dendritic epidermal T cells on skin wound healing. Front Immunol. (2018) 9:1099. doi: 10.3389/fimmu.2018.01099

8. Kohlgruber AC, Gal-Oz ST, LaMarche NM, Shimazaki M, Duquette D, Koay $\mathrm{HF}$, et al. gammadelta $\mathrm{T}$ cells producing interleukin-17A regulate adipose regulatory $\mathrm{T}$ cell homeostasis and thermogenesis. Nat Immunol. (2018) 19:464-74. doi: 10.1038/s41590-018-0094-2

9. Cai Y, Xue F, Fleming C, Yang J, Ding C, Ma Y, et al. Differential developmental requirement and peripheral regulation for dermal Vgamma4 and Vgamma6T17 cells in health and inflammation. Nat Commun. (2014) 5:3986. doi: $10.1038 /$ ncomms4986

10. Zuberbuehler MK, Parker ME, Wheaton JD, Espinosa JR, Salzler HR, Park E, Ciofani M. The transcription factor c-Maf is essential for the commitment of IL-17-producing gammadelta T cells. Nat Immunol. (2019) 20:73-85. doi: 10.1038/s41590-018-0274-0

11. Rei M, Pennington DJ, Silva-Santos B. The emerging Protumor role of gammadelta T lymphocytes. implications for cancer immunotherapy. Cancer Res. (2015) 75:798-802. doi: 10.1158/0008-5472.CAN-14-3228 
12. Ma S, Cheng Q, Cai Y, Gong H, Wu Y, Yu X, et al. IL-17A produced by gammadelta $\mathrm{T}$ cells promotes tumor growth in hepatocellular carcinoma. Cancer Res. (2014) 74:1969-82. doi: 10.1158/0008-5472.CAN-13-2534

13. Hu G, Wu P, Cheng $\mathrm{P}$, Zhang Z, Wang Z, Yu X, et al. Tumorinfiltrating $\mathrm{CD} 39(+)$ gammadeltaTregs are novel immunosuppressive $\mathrm{T}$ cells in human colorectal cancer. Oncoimmunology. (2017) 6:e1277305. doi: 10.1080/2162402X.2016.1277305

14. Peng G, Wang HY, Peng W, Kiniwa Y, Seo KH, Wang RF. Tumor-infiltrating gammadelta $\mathrm{T}$ cells suppress $\mathrm{T}$ and dendritic cell function via mechanisms controlled by a unique toll-like receptor signaling pathway. Immunity. (2007) 27:334-48. doi: 10.1016/j.immuni.2007.05.020

15. Wu D, Wu P, Qiu F, Wei Q, Huang J. Human gammadeltaT-cell subsets and their involvement in tumor immunity. Cell Mol Immunol. (2017) 14:24553. doi: $10.1038 / \mathrm{cmi} .2016 .55$

16. Wu P, Wu D, Ni C, Ye J, Chen W, Hu G, et al. gammadeltaT17 cells promote the accumulation and expansion of myeloid-derived suppressor cells in human colorectal cancer. Immunity. (2014) 40:785-800. doi: 10.1016/j.immuni.2014.03.013

17. McAllister F, Bailey JM, Alsina J, Nirschl CJ, Sharma R, Fan H, et al. Oncogenic Kras activates a hematopoietic-to-epithelial IL-17 signaling axis in preinvasive pancreatic neoplasia. Cancer Cell. (2014) 25:62137. doi: 10.1016/j.ccr.2014.03.014

18. Jin C, Lagoudas GK, Zhao C, Bullman S, Bhutkar A, Hu B, et al. Commensal microbiota promote lung cancer development via $\gamma \delta \mathrm{T}$ cells. Cell. (2019) 176:998-1013. doi: 10.1016/j.cell.2018.12.040

19. Daley D, Zambirinis CP, Seifert L, Akkad N, Mohan N, Werba $\mathrm{G}$, et al. gammadelta $\mathrm{T}$ Cells Support Pancreatic Oncogenesis by Restraining alphabeta T Cell Activation. Cell. (2016) 166:148599.e1415. doi: 10.1016/j.cell.2016.07.046

20. Wu Y, Kyle-Cezar F, Woolf RT, Naceur-Lombardelli C, Owen J, Biswas D, et al. An innate-like Vdeltal(+) gammadelta $\mathrm{T}$ cell compartment in the human breast is associated with remission in triple-negative breast cancer. Sci Transl Med. (2019) 11:eaax9364. doi: 10.1126/scitranslmed.aax9364

21. Ivanov, II, McKenzie BS, Zhou L, Tadokoro CE, Lepelley A, Lafaille JJ, et al. The orphan nuclear receptor RORgammat directs the differentiation program of proinflammatory IL-17+ T helper cells. Cell. (2006) 126:112133. doi: $10.1016 /$ j.cell.2006.07.035

22. Barros-Martins J, Schmolka N, Fontinha D, Pires de Miranda M, Simas JP, Brok I, et al. Effector gammadelta $\mathrm{T}$ cell differentiation relies on master but not auxiliary Th cell transcription factors. J Immunol. (2016) 196:364252. doi: 10.4049/jimmunol.1501921

23. Raifer H, Mahiny AJ, Bollig N, Petermann F, Hellhund A, Kellner K, et al. Unlike alphabeta T cells, gammadelta T cells, LTi cells and NKT cells do not require IRF4 for the production of IL-17A and IL-22. Eur J Immunol. (2012) 42:3189-201. doi: 10.1002/eji.201142155

24. Cai Y, Xue F, Qin H, Chen X, Liu N, Fleming C, et al. Differential roles of the mTOR-STAT3 signaling in dermal gammadelta $\mathrm{T}$ cell effector function in skin inflammation. Cell Rep. (2019) 27:3034-48.e3035. doi: 10.1016/j.celrep.2019.05.019

25. Cua DJ, Tato CM. Innate IL-17-producing cells: the sentinels of the immune system. Nat Rev Immunol. (2010) 10:479-89. doi: 10.1038/nri2800

26. Agerholm R, Rizk J, Vinals MT, Bekiaris V. STAT3 but not STAT4 is critical for gammadeltaT17 cell responses and skin inflammation. EMBO Rep. (2019) 2019:e48647. doi: 10.15252/embr.201948647

27. Malhotra N, Narayan K, Cho OH, Sylvia KE, Yin C, Melichar H, et al. A network of high-mobility group box transcription factors programs innate interleukin-17 production. Immunity. (2013) 38:68193. doi: $10.1016 /$ j.immuni.2013.01.010

28. In TSH, Trotman-Grant A, Fahl S, Chen ELY, Zarin P, Moore AJ, et al. HEB is required for the specification of fetal IL-17-producing gammadelta T cells. Nat Commun. (2017) 8:2004. doi: 10.1038/s41467-017-02225-5

29. Lu Y, Cao X, Zhang X, Kovalovsky D. PLZF controls the development of fetal-derived IL-17+Vgamma6+ gammadelta T cells. J Immunol. (2015) 195:4273-81. doi: 10.4049/jimmunol.1500939

30. Martin B, Hirota K, Cua DJ, Stockinger B, Veldhoen M. Interleukin17-producing gammadelta $\mathrm{T}$ cells selectively expand in response to pathogen products and environmental signals. Immunity. (2009) 31:32130. doi: 10.1016/j.immuni.2009.06.020
31. Sutton CE, Lalor SJ, Sweeney CM, Brereton CF, Lavelle EC, Mills KH. Interleukin-1 and IL-23 induce innate IL-17 production from gammadelta $\mathrm{T}$ cells, amplifying Th17 responses and autoimmunity. Immunity. (2009) 31:331-41. doi: 10.1016/j.immuni.2009.08.001

32. Cai Y, Xue F, Quan C, Qu M, Liu N, Zhang Y, et al. A critical role of the IL-1beta-IL-1R signaling pathway in skin inflammation and psoriasis pathogenesis. J Invest Dermatol. (2019) 139:146-56. doi: 10.1016/j.jid.2018.07.025

33. Fleming C, Cai Y, Sun X, Jala VR, Xue F, Morrissey S, et al. Microbiotaactivated CD103(+) DCs stemming from microbiota adaptation specifically drive gammadeltaT17 proliferation and activation. Microbiome. (2017) 5:46. doi: 10.1186/s40168-017-0263-9

34. Powolny-Budnicka I, Riemann M, Tanzer S, Schmid RM, Hehlgans T, Weih F. RelA and RelB transcription factors in distinct thymocyte populations control lymphotoxin-dependent interleukin-17 production in gammadelta $\mathrm{T}$ cells. Immunity. (2011) 34:364-74. doi: 10.1016/j.immuni.2011.02.019

35. Mair F, Joller S, Hoeppli R, Onder L, Hahn M, Ludewig B, et al. The NFkappaB-inducing kinase is essential for the developmental programming of skin-resident and IL-17-producing gammadelta T cells. Elife. (2015) 4:e10087. doi: 10.7554/eLife.10087

36. Shibata K, Yamada $H$, Sato $T$, Dejima $T$, Nakamura $M$, Ikawa $\mathrm{T}$, et al. Notch-Hesl pathway is required for the development of IL-17-producing gammadelta $\mathrm{T}$ cells. Blood. (2011) 118:58693. doi: 10.1182/blood-2011-02-334995

37. Munoz-Ruiz M, Ribot JC, Grosso AR, Goncalves-Sousa N, Pamplona A, Pennington DJ, et al. TCR signal strength controls thymic differentiation of discrete proinflammatory gammadelta T cell subsets. Nat Immunol. (2016) 17:721-7. doi: $10.1038 /$ ni. 3424

38. Spidale NA, Sylvia K, Narayan K, Miu B, Frascoli M, Melichar HJ, et al. Interleukin-17-producing gammadelta $\mathrm{T}$ cells originate from $\mathrm{SOX} 13(+)$ progenitors that are independent of gammadeltaTCR signaling. Immunity. (2018) 49:857-72.e855. doi: 10.1016/j.immuni.2018.09.010

39. Muro R, Nitta $T$, Nakano $K$, Okamura $T$, Takayanagi $H$, Suzuki H. gammadeltaTCR recruits the Syk/PI3K axis to drive proinflammatory differentiation program. J Clin Invest. (2018) 128:415-26. doi: 10.1172/JCI95837

40. McKenzie DR, Comerford I, Silva-Santos B, McColl SR. The emerging complexity of gammadeltaT17 cells. Front Immunol. (2018) 9:796. doi: 10.3389/fimmu.2018.00796

41. Moens E, Brouwer M, Dimova T, Goldman M, Willems F, Vermijlen D. IL23R and TCR signaling drives the generation of neonatal Vgamma9Vdelta2 $\mathrm{T}$ cells expressing high levels of cytotoxic mediators and producing IFN-gamma and IL-17. J Leukoc Biol. (2011) 89:743-52. doi: 10.1189/jlb.0910501

42. Wilson RP, Ives ML, Rao G, Lau A, Payne K, Kobayashi M, et al. STAT3 is a critical cell-intrinsic regulator of human unconventional $\mathrm{T}$ cell numbers and function. J Exp Med. (2015) 212:855-64. doi: 10.1084/jem.20141992

43. Venken K, Jacques P, Mortier C, Labadia ME, Decruy T, Coudenys J, et al. RORgammat inhibition selectively targets IL-17 producing iNKT and gammadelta-T cells enriched in Spondyloarthritis patients. Nat Commun. (2019) 10:9. doi: 10.1038/s41467-018-07911-6

44. Ness-Schwickerath KJ, Jin C, Morita CT. Cytokine requirements for the differentiation and expansion of IL-17A- and IL-22producing human Vgamma2Vdelta2 $\mathrm{T}$ cells. J Immunol. (2010) 184:7268-80. doi: 10.4049/jimmunol.1000600

45. Caccamo N, La Mendola C, Orlando V, Meraviglia S, Todaro M, Stassi G, et al. Differentiation, phenotype, and function of interleukin17-producing human Vgamma9Vdelta2 T cells. Blood. (2011) 118:12938. doi: 10.1182/blood-2011-01-331298

46. Michalek RD, Gerriets VA, Jacobs SR, Macintyre AN, MacIver NJ, Mason EF, et al. Cutting edge. distinct glycolytic and lipid oxidative metabolic programs are essential for effector and regulatory CD4+ T cell subsets. J Immunol. (2011) 186:3299-303. doi: 10.4049/jimmunol.1003613

47. Dimeloe S, Burgener AV, Grahlert J, Hess C. T-cell metabolism governing activation, proliferation and differentiation; a modular view. Immunology. (2017) 150:35-44. doi: 10.1111/imm.12655

48. Geltink RIK, Kyle RL, Pearce EL. Unraveling the complex interplay between T cell metabolism and function. Annu Rev Immunol. (2018) 36:46188. doi: 10.1146/annurev-immunol-042617-053019 
49. Pollizzi KN, Powell JD. Integrating canonical and metabolic signalling programmes in the regulation of T cell responses. Nat Rev Immunol. (2014) 14:435-46. doi: 10.1038/nri3701

50. Shi LZ, Wang R, Huang G, Vogel P, Neale G, Green DR, Chi H. HIF1alphadependent glycolytic pathway orchestrates a metabolic checkpoint for the differentiation of TH17 and Treg cells. J Exp Med. (2011) 208:136776. doi: 10.1084/jem.20110278

51. Zeng H, Yang K, Cloer C, Neale G, Vogel P, Chi H. mTORC1 couples immune signals and metabolic programming to establish $\mathrm{T}(\mathrm{reg})$-cell function. Nature. (2013) 499:485-90. doi: 10.1038/nature12297

52. Waickman AT, Powell JD. mTOR, metabolism, and the regulation of T-cell differentiation and function. Immunol Rev. (2012) 249:4358. doi: 10.1111/j.1600-065X.2012.01152.x

53. Mensurado S, Rei M, Lanca T, Ioannou M, Goncalves-Sousa N, Kubo $\mathrm{H}$, et al. Tumor-associated neutrophils suppress pro-tumoral IL-17+ gammadelta T cells through induction of oxidative stress. PLoS Biol. (2018) 16:e2004990. doi: 10.1371/journal.pbio.2004990

54. Wang R, Dillon CP, Shi LZ, Milasta S, Carter R, Finkelstein $\mathrm{D}$, et al. The transcriptionfactor Myc controls metabolic reprogramming upon $\mathrm{T}$ lymphocyte activation. Immunity. 35:871-82. doi: 10.1016/j.immuni.2011.09.021

55. Cheng M, Qian L, Shen G, Bian G, Xu T, Xu W, et al. Microbiota modulate tumoral immune surveillance in lung through a gammadeltaT17 immune cell-dependent mechanism. Cancer Res. (2014) 74:4030-41. doi: 10.1158/0008-5472.CAN-1 3-2462

Conflict of Interest: The authors declare that the research was conducted in the absence of any commercial or financial relationships that could be construed as a potential conflict of interest.

Copyright (C) 2019 Chen, Morrissey, Chen and Yan. This is an open-access article distributed under the terms of the Creative Commons Attribution License (CC BY). The use, distribution or reproduction in other forums is permitted, provided the original author(s) and the copyright owner(s) are credited and that the original publication in this journal is cited, in accordance with accepted academic practice. No use, distribution or reproduction is permitted which does not comply with these terms. 